\title{
ON MULTIPLICATIVE COMPOSITIONS OF INTEGERS
}

\author{
HUGH L. MONTGOMERY AND GÉRALD TENENBAUM
}

Dedicated to the memory of K. F. Roth

Abstract. We consider an arithmetic function defined independently by John G. Thompson and Greg Simay, with particular attention to its mean value, its maximal size, and the analytic nature of its Dirichlet series generating function.

$\S 1$. Introduction. In combinatorics, a composition of a positive integer $n$ is a representation of $n$ as a sum of positive integers in which the order of the summands matters. For example, $4=4=3+1=1+3=2+2=2+1+1=$ $1+2+1=1+1+2=1+1+1+1$. Thus there are 8 compositions of 4 . It is easy to see that in general there are exactly $2^{n-1}$ compositions of $n$. Analogously, we let $c(n)$ denote the number of representations of $n$ as a product of primes, in which the order of the factors matters. Thus $c(p)=1, c\left(p_{1} p_{2}\right)=2$, and $c\left(p_{1}^{2} p_{2}\right)=3$, and so $c(n)$ is not a multiplicative function. In general, if $n=$ $p_{1}^{\nu_{1}} p_{2}^{\nu_{2}} \cdots p_{r}^{v_{r}}$ is the canonical factorization of $n$ and $\Omega(n)=\sum_{j} v_{j}$, then $c(n)$ is the multinomial coefficient

$$
c(n)=\left(\begin{array}{c}
\Omega(n) \\
v_{1}, \ldots, v_{r}
\end{array}\right)=\frac{\Omega(n) !}{\prod_{i=1}^{r} v_{i} !} .
$$

John G. Thompson, in the course of some of his (as yet unpublished) research on the Riemann zeta function, defined the Dirichlet series

$$
D(s)=\sum_{n \geqslant 1} \frac{c(n)}{n^{s}}
$$

and asked for its abscissa of convergence, while Greg Simay in a private communication noted the combinatorial interpretation of the coefficients $c(n)$ and inquired about their asymptotic mean value.

THEOREM 1.1. For $\sigma:=\Re s>1$, and non-negative integers $k$, let

$$
G_{k}(s)=\sum_{p} \frac{(\log p)^{k}}{p^{s}} .
$$

Let $\sigma_{0} \approx 1.399433329$ denote the unique real number $>1$ such that

$$
G_{0}\left(\sigma_{0}\right)=1
$$

Received 28 February 2017.

MSC (2010): 11N37, 11N56 (primary), 11M41 (secondary).

First author supported in part by National Science Foundation Grant DMS-063529. 
With $c(n)$ and $D(s)$ defined as in (1) and (2), we have

$$
D(s)=\frac{1}{1-G_{0}(s)}
$$

for $\sigma>\sigma_{0}$, and $\sigma_{0}$ is the abscissa of convergence of this Dirichlet series.

Proof. Suppose that $\sigma>1$. By the multinomial theorem,

$$
G_{0}(s)^{m}=\sum_{\substack{n \geqslant 1 \\ \Omega(n)=m}} \frac{c(n)}{n^{s}} .
$$

We sum this over $m=0,1,2, \ldots$ to obtain (2). Since $\lim _{\sigma \rightarrow \sigma_{0}+} D(\sigma)=+\infty$, and the coefficients are non-negative, it follows that $D(s)$ does not converge at $s=\sigma_{0}$. Hence $\sigma_{0}$ is the abscissa of convergence of $D(s)$.

By the Möbius inversion formula it is evident that, for $\sigma:=\Re s>1$,

$$
\begin{aligned}
& G_{0}(s)=\sum_{d \geqslant 1} \frac{\mu(d)}{d} \log \zeta(d s), \\
& G_{1}(s)=-\sum_{d \geqslant 1} \mu(d) \frac{\zeta^{\prime}}{\zeta}(d s),
\end{aligned}
$$

and these series actually converge for $\sigma>0$ if the pole and zeros of the zeta-function are suitably avoided. Moreover $G_{1}(s)$ may be continued to the half-plane $\sigma>0$ as a meromorphic function with poles of order 1. Since $\zeta(s)$ can be evaluated by the Euler-MacLaurin formula (or by the RiemannSiegel formula), one can calculate $G_{0}(s)$ without a detailed knowledge of the distribution of prime numbers. The function $D(s)$ has poles at points where $G_{0}(s)=1$; the abscissæ of such points are dense in $\left(1, \sigma_{0}\right)$. In addition, $D(s)$ has a transcendental singularity at points of the form $1 / d$ or $\varrho / d$ for squarefree $d$, where $\varrho$ runs through the non-trivial zeros of the zeta-function. By slitting the complex plane suitably one may continue $D(s)$ to $\sigma>0$, but the imaginary axis is a natural boundary for $D(s)$, since Landau and Walfisz [4] showed that $\sigma=0$ is a natural boundary of $G_{0}(s)=1-1 / D(s)$.

§. The summatory function. With $c(n)$ as in (1), let

$$
C(x)=\sum_{n \leqslant x} c(n) .
$$

By a basic theorem concerning Dirichlet series (as in Montgomery and Vaughan [5, Theorem 1.3] or Tenenbaum [7, Theorem II.1.13]) it follows from Theorem 1.1 that

$$
\limsup _{x \rightarrow \infty} \frac{\log C(x)}{\log x}=\sigma_{0} .
$$


As $c(n) \geqslant 0$ for all $n$, and

$$
D(s)-\frac{1}{G_{1}\left(\sigma_{0}\right)\left(s-\sigma_{0}\right)}
$$

is continuous in the closed half-plane $\sigma \geqslant \sigma_{0}$, it follows by the Wiener-Ikehara theorem (as discussed, for example, in Montgomery and Vaughan [5, §8.3]) that

$$
C(x) \sim \frac{x^{\sigma_{0}}}{\sigma_{0} G_{1}\left(\sigma_{0}\right)}
$$

as $x \rightarrow \infty$. Here we see that the average size of $c(n)$ is $c n^{\sigma_{0}-1}$, but

$$
c(n) \leqslant \Omega(n)^{\Omega(n)}
$$

for all $n$, so

$$
c(n) \leqslant(\log n)^{(1+\varepsilon) \log _{3} n}
$$

for almost all $n$ (in the sense of natural density), where, here and in the sequel, $\log _{k}$ denotes the $k$-fold iterated logarithm.

The estimate (8) can be made quantitative by using further information about $D(s)$. For example, suppose we put $\alpha:=(\log 2) / \log 3$. Since $\alpha$ is irrational, it follows that there is no $\tau \neq 0$ such that $2^{\mathrm{i} \tau}=3^{\mathrm{i} \tau}=1$. We note that Salykhov [6] has given a measure of the irrationality of $\alpha$. In the improved from of $\mathrm{Wu}$ and Wang [8], it asserts that if $\delta=4.117$, then

$$
\|q \alpha\| \gg 1 / q^{\delta} \quad\left(q \in \mathbb{Z}^{+}\right)
$$

Here $\|\vartheta\|$ denotes the distance from the real number $\vartheta$ to the set of integers. We note that if $s:=\sigma+\mathrm{i} \tau$ with $\sigma \geqslant 0$, then

$$
\begin{aligned}
\left|1-G\left(\sigma_{0}+s\right)\right| & =\left|\sum_{p} p^{-\sigma_{0}}\left(1-p^{-s}\right)\right| \geqslant \Re \sum_{p} p^{-\sigma_{0}}\left(1-p^{-s}\right) \\
& \gg \sum_{p} p^{-\sigma_{0}} \sin ^{2}\left(\frac{1}{2} \tau \log p\right) \\
& \geqslant \sin ^{2}\left(\frac{1}{2} \tau \log 2\right)+\sin ^{2}\left(\frac{1}{2} \tau \log 3\right) .
\end{aligned}
$$

On putting $\vartheta:=\tau(\log 3) /(2 \pi)$, we find that this is

$$
=\sin (\pi \alpha \vartheta)^{2}+(\sin \pi \vartheta)^{2} \asymp\|\alpha \vartheta\|^{2}+\|\vartheta\|^{2} .
$$

If $\tau_{0}<|\tau| \ll 1$, the above expression is $\gg 1$. Otherwise there is some $q \in \mathbb{Z}^{+}$ such that $\varepsilon:=\min (|\vartheta-q|,|\vartheta+q|) \leqslant \frac{1}{2}$. If $\varepsilon \leqslant \kappa / q^{\delta}$ with sufficiently small, absolute $\kappa>0$ then, by (9), we have

$$
\|\alpha \vartheta\| \geqslant\|\alpha q\|-\frac{\alpha \kappa}{q^{\delta}} \gg \frac{1}{q^{\delta}} .
$$


If $\varepsilon>\kappa / q^{\delta}$, then $\|\vartheta\| \gg 1 / q^{\delta}$. Thus the right-hand side of (10) is $\gg 1 / q^{2 \delta}$ for all $\vartheta \geqslant 1$. Since $q \asymp \vartheta \asymp \tau$, we deduce that

$$
D\left(\sigma_{0}+s\right) \ll \tau^{2 \delta}, \quad D^{\prime}\left(\sigma_{0}+s\right) \ll \tau^{2 \delta} \quad(\sigma \geqslant 0, \tau \geqslant 1) .
$$

Inserting this information into the effective Ikehara-Ingham-Delange Tauberian theorem of Tenenbaum [7, Theorem II.7.13], we find that, if $K:=1 /(4 \delta+1) \approx$ 0.05724 , then

$$
C(x)=\frac{x^{\sigma_{0}}}{\sigma_{0} G_{1}\left(\sigma_{0}\right)}\left\{1+O\left(\frac{1}{(\log x)^{K}}\right)\right\}
$$

as $x \rightarrow \infty$. Although the error term here is not as small as we would like, it raises the question as to whether lower bounds for linear forms in logarithms of small primes might be used to obtain a better error term, possibly even a very good error term.

Due to the many singularities of $D(s)$, the asymptotic estimate (12) cannot be sharpened so as to save a power of $x$. Indeed, since (i) the $c(n)$ are nonnegative, (ii) $D(s)$ is regular on the real axis in the interval $\left(1, \sigma_{0}\right)$, and (iii) $D(s)$ has poles with abscissæ $>\sigma_{0}-\varepsilon$, it follows by the Phragmén-Landau theorem (cf. Montgomery and Vaughan [5, §15.1] or Tenenbaum [7, Theorems 1.11 and 1.12]) that

$$
C(x)-\frac{x^{\sigma_{0}}}{\sigma_{0} G_{1}\left(\sigma_{0}\right)}=\Omega_{ \pm}\left(x^{\sigma_{0}-\varepsilon}\right)
$$

for all $\varepsilon>0$.

§3. A pole-free region for $D(s)$. Tauberian theorems are easy to employ because they do not require any knowledge of an analytic continuation for the Dirichlet series involved. As pointed out by Karamata [3], the corresponding drawback is that, when available, their remainder terms are necessarily weak. We now determine a pole-free region for $D(s)$ in order to obtain, via contour integration, a better error term in the asymptotic estimate for $C(x)$.

For every $\tau \geqslant 10$, there exists an $X_{0}(\tau)$ such that if $x \geqslant X_{0}(\tau)$, then

$$
\sum_{x \leqslant n \leqslant 3 x} \Lambda(n)\{1-\cos (\tau \log n)\} \geqslant \frac{1}{4} x .
$$

Of course, by the prime number theorem the left-hand side is $\sim 2 x$ as $x$ tends to infinity. The issue is how large must we make $x$ in order to be assured of obtaining a positive fraction of this amount.

Lemma 3.1. Let $X_{0}(\tau)$ be defined as in (14). There exists an absolute constant $c>0$ such that

$$
X_{0}(\tau) \leqslant \exp \left\{c(\log \tau)^{2 / 3}\left(\log _{2} \tau\right)^{4 / 3}\right\} .
$$

If the Riemann Hypothesis $(R H)$ is true, then

$$
X_{0}(\tau) \ll(\log \tau)^{2} .
$$


Proof. We may clearly assume $\tau$ is arbitrarily large. Write

$$
\begin{array}{ll}
\mathscr{L}(z):=\exp \left\{\frac{(\log z)^{3 / 5}}{\left(\log _{2} z\right)^{1 / 5}}\right\} & (z \geqslant 3), \\
\beta(T):=(\log T)^{-2 / 3}\left(\log _{2} T\right)^{-1 / 3} & (T \geqslant 3) .
\end{array}
$$

Using the Vinogradov-Korobov zero-free region $\sigma \geqslant 1-c_{1} \beta(T)$ for the zeta function with $s=\sigma+i \tau, T:=3+|\tau|$, where $c_{1}$ is an absolute constant (see e.g. [2, Ch. 6]), and the validity of the bound $\zeta^{\prime}(s) / \zeta(s) \ll \log T$ in the same region, we may employ standard complex integration to get, for $x \geqslant 3, \tau \in \mathbb{R}$, $\log T \leqslant(\log x)^{3 / 2} /\left(\log _{2} x\right)^{2}$,

$$
\sum_{n \leqslant x} \frac{\Lambda(n)}{n^{i \tau}} \ll x^{1-c_{2} \beta(T)}+\frac{x}{T}+\frac{x}{\mathscr{L}(x)^{c_{2}}},
$$

where $c_{2}$ is absolute. We omit the details which are very similar to those in the proof of [7, Lemma III.5.16]. This immediately implies (15).

Assuming RH, we note that

$$
\begin{aligned}
& \sum_{x \leqslant n \leqslant 3 x} \Lambda(n)\{1-\cos (\tau \log n)\} \\
& \geqslant \frac{1}{x} \sum_{x \leqslant n \leqslant 3 x}(x-|n-2 x|) \Lambda(n)\{1-\cos (\tau \log n)\} \\
& =x+o(x)-\frac{1}{x} \sum_{x \leqslant n \leqslant 3 x}(x-|n-2 x|) \Lambda(n) \cos (\tau \log n) .
\end{aligned}
$$

Thus it suffices to show that

$$
\left|\sum_{x \leqslant n \leqslant 3 x}(x-|n-2 x|) \frac{\Lambda(n)}{n^{\mathrm{i} \tau}}\right| \leqslant \frac{1}{5} x^{2} .
$$

The above sum is

$$
\begin{aligned}
& =\frac{-1}{2 \pi \mathrm{i}} \int_{2-\mathrm{i} \infty}^{2+\mathrm{i} \infty} \frac{\zeta^{\prime}}{\zeta}(s+\mathrm{i} \tau) \frac{(3 x)^{s+1}-2(2 x)^{s+1}+x^{s+1}}{s(s+1)} d s \\
& \ll \frac{x^{2}}{\tau^{2}}+\sum_{\gamma} \frac{x^{\beta+1}}{(\gamma-\tau)^{2}+1} .
\end{aligned}
$$

Assuming RH, the sum over $\gamma$ is $\ll x^{3 / 2} \log \tau$ since the number of $\gamma$ in $[u, u+1]$ is $\ll \log (3+|u|)$. The desired bound follows for all $x \geqslant X_{0}(\tau)$ by selecting $X_{0}(\tau)=C(\log \tau)^{2}$ with $C$ a sufficiently large absolute constant.

THEOREM 3.2. There exists an absolute constant $c>0$ such that if $\tau \geqslant 10$, then

$$
D(s) \ll \exp \left\{c(\log \tau)^{2 / 3}\left(\log _{2} \tau\right)^{4 / 3}\right\}
$$


uniformly for

$$
\sigma \geqslant \sigma_{0}-\exp \left\{-c(\log \tau)^{2 / 3}\left(\log _{2} \tau\right)^{4 / 3}\right\} .
$$

Assuming $R H$, if $\tau \geqslant 10$, then

$$
D(s) \ll(\log \tau)^{4 / 5}
$$

uniformly for

$$
\sigma \geqslant \sigma_{0}-\frac{1}{(\log \tau)^{4 / 5}}
$$

Proof. Since $\sum_{p} p^{-\sigma_{0}}=1$, it follows that

$$
\begin{aligned}
1-\mathfrak{R} \sum_{p} p^{-s} & =\sum_{p} p^{-\sigma_{0}}-\sum_{p} p^{-\sigma} \cos (t \log p) \\
& =\sum_{p} p^{-\sigma}\{1-\cos (\tau \log p)\}-\sum_{p}\left(p^{-\sigma}-p^{-\sigma_{0}}\right) .
\end{aligned}
$$

Here the last term is

$$
G_{0}(\sigma)-G_{0}\left(\sigma_{0}\right)=-\int_{\sigma}^{\sigma_{0}} G_{0}^{\prime}(\alpha) d \alpha=\int_{\sigma}^{\sigma_{0}} G_{1}(\alpha) d \alpha .
$$

Now $G_{1}$ is a decreasing function, and $G_{1}(1.3)=2.475639<2.5$, so if $\sigma \geqslant 1.3$, then the above is $\leqslant \frac{5}{2}\left(\sigma_{0}-\sigma\right)$. Let $X_{0}(\tau)$ be defined as in (14). Then the expression (22) is

$$
\geqslant \frac{c X_{0}(\tau)^{1-\sigma}}{\log X_{0}(\tau)}-\frac{5}{2}\left(\sigma_{0}-\sigma\right)
$$

We now suppose that $\sigma$ is close enough to $\sigma_{0}$ to ensure that the second term above is at most one half the first term. For such $\sigma$ we have a lower bound for the real part of $1-G_{0}(s)=1 / D(s)$, and hence an upper bound for $|D(s)|$. To complete the argument it suffices to substitute the upper bounds for $X_{0}(\tau)$ derived in Lemma 3.1.

§4. A stronger quantitative mean value theorem.

THEOREM 4.1. There is a constant $a>0$ such that

$$
C(x)=\frac{x^{\sigma_{0}}}{\sigma_{0} G_{1}\left(\sigma_{0}\right)}\left(1+O\left(\exp \left\{-a\left(\log _{2} x\right)^{3 / 2} /\left(\log _{3} x\right)^{2}\right\}\right)\right)
$$

uniformly for $x \geqslant 3$. Assuming $R H$,

$$
C(x)=\frac{x^{\sigma_{0}}}{\sigma_{0} G_{1}\left(\sigma_{0}\right)}\left(1+O\left(\exp \left\{-a(\log x)^{5 / 9}\right\}\right)\right) .
$$


Proof. Let

$$
C_{1}(x)=\sum_{n \leqslant x}(x-n) c(n)=\frac{1}{2 \pi \mathrm{i}} \int_{\alpha-\mathrm{i} \infty}^{\alpha+\mathrm{i} \infty} D(s) \frac{x^{s+1}}{s(s+1)} d s .
$$

We move the path of integration to form a rectilinear contour $\mathscr{C}$ from $\sigma_{0}-\mathrm{i} \infty$ to $\sigma_{0}-\mathrm{i} T$ to $\sigma_{0}-\delta-\mathrm{i} T$ to $\sigma_{0}-\delta+\mathrm{i} T$ to $\sigma_{0}+\mathrm{i} T$ to $\sigma_{0}+\mathrm{i} \infty$ where

$$
\delta:=\exp \left\{-c(\log T)^{2 / 3}\left(\log _{2} T\right)^{4 / 3}\right\}, \quad T:=\exp \left\{a\left(\log _{2} x\right)^{3 / 2}\left(\log _{3} x\right)^{-2}\right\} .
$$

Thus the above is

$$
=\frac{x^{\sigma_{0}+1}}{\sigma_{0}\left(\sigma_{0}+1\right) G_{1}\left(\sigma_{0}\right)}+\frac{1}{2 \pi \mathrm{i}} \int_{\mathscr{C}} D(s) \frac{x^{s+1}}{s(s+1)} d s .
$$

We note that

$$
c(\log T)^{2 / 3}\left(\log _{2} T\right)^{4 / 3} \sim c a^{2 / 3}\left(\frac{3}{2}\right)^{4 / 3} \log _{2} x .
$$

We take $a$ to be small enough to ensure that the right-hand side above is $<\frac{1}{3} \log _{2} x$. Thus

$$
\begin{aligned}
& \delta \geqslant(\log x)^{-1 / 3}, \quad x^{-\delta}<\exp \left\{-(\log x)^{2 / 3}\right\}, \\
& D\left(\sigma_{0}-\delta+\mathrm{i} \tau\right) \ll(\log x)^{1 / 3} \quad(-T \leqslant \tau \leqslant T) .
\end{aligned}
$$

Consequently,

$$
\begin{gathered}
\int_{\sigma_{0}-\delta-\mathrm{i} T}^{\sigma_{0}-\delta+\mathrm{i} T} \ll x^{\sigma_{0}+1} \exp \left\{-(\log x)^{2 / 3}\right\}(\log x)^{1 / 3}, \\
\int_{\sigma_{0}-\delta+\mathrm{i} T}^{\sigma_{0}+\mathrm{i} T} \ll \frac{x^{\sigma_{0}+1}}{T^{2}}, \quad \int_{\sigma_{0}+\mathrm{i} T}^{\sigma_{0}+\mathrm{i} \infty} \ll \frac{x^{\sigma_{0}+1}}{T^{2}}(\log x)^{1 / 3} .
\end{gathered}
$$

Thus

$$
C_{1}(x)=\frac{x^{\sigma_{0}+1}}{\sigma_{0}\left(\sigma_{0}+1\right) G_{1}\left(\sigma_{0}\right)}+O\left(x^{\sigma_{0}+1} \exp \left(-b\left(\log _{2} x\right)^{3 / 2}\left(\log _{3} x\right)^{-2}\right)\right) .
$$

Since $c(n) \geqslant 0$ for all $n$, it follows that

$$
\frac{C_{1}(x)-C_{1}(x-h)}{h} \leqslant C(x) \leqslant \frac{C_{1}(x+h)-C_{1}(x)}{h} .
$$

We note that

$$
\frac{(x+h)^{\sigma_{0}+1}-x^{\sigma_{0}+1}}{h\left(\sigma_{0}+1\right)}=\frac{1}{h} \int_{x}^{x+h} u^{\sigma_{0}} d u \leqslant(x+h)^{\sigma_{0}}=x^{\sigma_{0}}+O\left(h x^{\sigma_{0}-1}\right) .
$$

Hence

$$
C(x) \leqslant \frac{x^{\sigma_{0}+1}}{\sigma_{0} G_{1}\left(\sigma_{0}\right)}+O\left(h x^{\sigma_{0}-1}\right)+O\left(h^{-1} x^{\sigma_{0}+1} \exp \left\{-b\left(\log _{2} x\right)^{3 / 2}\left(\log _{3} x\right)^{-2}\right\}\right) .
$$


We take $h=x \exp \left(-\frac{1}{2} b\left(\log _{2} x\right)^{3 / 2}\left(\log _{3} x\right)^{-2}\right)$ to obtain the upper bound part of our estimate. The lower bound is derived similarly.

When we assume RH we argue similarly, with

$$
T:=\exp \left\{(\log x)^{5 / 9}\right\}, \quad \delta:=(\log T)^{-4 / 5}=(\log x)^{-4 / 9} .
$$

Thus $x^{-\delta}=\exp \left((\log x)^{-5 / 9}\right)=1 / T$.

§5. Large values of $c(n)$. From (12) or Theorem 4.1 it follows that $c(n)=o\left(n^{\sigma_{0}}\right)$ as $n \rightarrow \infty$. To complete our discussion we show that there are $n$ for which $c(n)$ is nearly this large.

THEOREM 5.1. Let $\sigma_{0}$ be defined as in (4). There is an absolute constant $C>0$ such that

$$
c(n)>n^{\sigma_{0}} \exp \left\{-C(\log n)^{1 / \sigma_{0}}\right\}
$$

for infinitely many integers $n$.

From the above it is evident that

$$
C(x)-\frac{c}{\sigma_{0}} x^{\sigma_{0}}=\Omega\left(x^{\sigma_{0}} \exp \left\{-C(\log x)^{1 / \sigma_{0}}\right\}\right) .
$$

Here the order of magnitude is more precise than in (13), but the above does not guarantee that the error becomes large in both signs.

Numerical studies indicate that large values of $c(n)$ occur only when $n$ is quite large. For example, the least $n$ for which $c(n)>n$ is

$$
n=326918592000=2^{9} \cdot 3^{6} \cdot 5^{3} \cdot 7^{2} \cdot 11 \cdot 13,
$$

for which

$$
c(n)=358500542400=\left(\begin{array}{c}
22 \\
9,6,3,2,1,1
\end{array}\right) .
$$

Proof. Let $y$ be a large real number, and consider $n=\prod_{p \leqslant y} p^{v(p)}$ where the $v(p)$ are of the form

$$
v(p)=\left\lfloor\frac{y^{\sigma}}{p^{\sigma}}\right\rfloor .
$$

Here $\sigma>1$ is chosen later, but we assume throughout our calculations that $\sigma$ is bounded. For brevity we put

$$
S_{k}(\sigma)=\sum_{p \leqslant y} \frac{(\log p)^{k}}{p^{\sigma}} .
$$

First we note that

$$
\log n=\sum_{p \leqslant y} v(p) \log p=y^{\sigma} S_{1}(\sigma)+O(y) .
$$


Similarly,

$$
\Omega(n)=\sum_{p \leqslant y} v(p)=y^{\sigma} S_{0}(\sigma)+O(y / \log y) .
$$

Hence

$$
\log \Omega(n)=\sigma \log y+\log S_{0}(\sigma)+O\left(y^{1-\sigma} / \log y\right) .
$$

We appeal to Stirling's formula in the crude form

$$
\log m !=m \log m-m+O(\log 2 m) .
$$

Thus

$$
\begin{aligned}
\log \{\Omega(n) !\}= & \left\{y^{\sigma} S_{0}(\sigma)+O\left(\frac{y}{\log y}\right)\right\}\left\{\sigma \log y+\log S_{0}(\sigma)+O\left(\frac{y^{1-\sigma}}{\log y}\right)\right\} \\
& -y^{\sigma} S_{0}(\sigma)+O\left(\frac{y}{\log y}\right) \\
= & \sigma S_{0}(\sigma) y^{\sigma} \log y+S_{0}(\sigma) y^{\sigma} \log S_{0}(\sigma)-S_{0}(\sigma) y^{\sigma}+O(y) .
\end{aligned}
$$

From (31) we also find that

$$
\begin{aligned}
\log \{v(p) !\} & =\left\{\frac{y^{\sigma}}{p^{\sigma}}+O(1)\right\}\left\{\sigma \log \left(\frac{y}{p}\right)+O\left(\frac{p^{\sigma}}{y^{\sigma}}\right)\right\}-\frac{y^{\sigma}}{p^{\sigma}}+O\left(\log \frac{2 y}{p}\right) \\
& =\frac{\sigma y^{\sigma} \log (y / p)}{p^{\sigma}}-\frac{y^{\sigma}}{p^{\sigma}}+O\left(\log \frac{2 y}{p}\right) .
\end{aligned}
$$

Hence

$$
\sum_{p \leqslant y} \log \{v(p) !\}=\sigma S_{0}(\sigma) y^{\sigma} \log y-\sigma S_{1}(\sigma) y^{\sigma}-S_{0}(\sigma) y^{\sigma}+O(y / \log y) .
$$

On combining this with (32) we deduce that

$$
\log c(n)=\left\{\sigma S_{1}(\sigma)+S_{0}(\sigma) \log S_{0}(\sigma)\right\} y^{\sigma}+O(y) .
$$

From (28) we deduce that this is

$$
=f(\sigma) \log n+O\left((\log n)^{1 / \sigma}\right)
$$

where

$$
f(\sigma)=\sigma+\frac{S_{0}(\sigma) \log S_{0}(\sigma)}{S_{1}(\sigma)} .
$$

Since $S_{k}^{\prime}(\sigma)=-S_{k+1}(\sigma)$, we find that

$$
\begin{aligned}
f^{\prime}(\sigma) & =1-\log S_{0}(\sigma)-1+\frac{S_{0}(\sigma) S_{2}(\sigma)}{S_{1}(\sigma)^{2}} \log S_{0}(\sigma) \\
& =\left(\frac{S_{0}(\sigma) S_{2}(\sigma)}{S_{1}(\sigma)^{2}}-1\right) \log S_{0}(\sigma) .
\end{aligned}
$$


Now $S_{1}(\sigma)^{2} \leqslant S_{0}(\sigma) S_{2}(\sigma)$ by Cauchy's inequality, and indeed $S_{1}(\sigma)^{2}<$ $S_{0}(\sigma) S_{2}(\sigma)$ since the summands are not proportional. Thus $f^{\prime}(\sigma)>0$ when $S_{0}(\sigma)>1$ and $f^{\prime}(\sigma)<0$ when $S_{0}(\sigma)<1$. Hence $f(\sigma)$ is maximized by choosing $\sigma$ so that $S_{0}(\sigma)=1$. This $\sigma$ depends on $y$, but is very close to $\sigma_{0}$. We obtain the same result — and the estimates are easier-if we take the slightly inferior choice $\sigma=\sigma_{0}$. Since

$$
\sum_{p>y} \frac{1}{p^{\sigma_{0}}} \sim \frac{y^{1-\sigma_{0}}}{\left(\sigma_{0}-1\right) \log y}
$$

it follows that

$$
\log S_{0}\left(\sigma_{0}\right) \ll \frac{y^{1-\sigma_{0}}}{\log y} \ll \frac{(\log n)^{1 / \sigma_{0}-1}}{\log _{2} n} .
$$

We now obtain the stated result by taking $\sigma=\sigma_{0}$ in (33).

Although the choice $\sigma=\sigma_{0}$ suffices asymptotically, it may still be the case in numerical studies with $y$ of moderate size that better results are obtained by choosing $\sigma$ so that $S_{0}(\sigma)=1$.

The estimate (28) can be refined: with more care it can be shown that

$$
\sum_{p \leqslant y}\left\lfloor\frac{y^{\sigma}}{p^{\sigma}}\right\rfloor \log p=y^{\sigma} S_{1}(\sigma)+\left\{\zeta\left(\frac{1}{\sigma}\right)+\frac{1}{\sigma-1}\right\} y+O\left(y \mathrm{e}^{-c \sqrt{\log y}}\right) .
$$

However, this does not seem to lead to a sharper result in the present context.

\section{References}

1. G. Freud, Restglied eines Tauberschen Satzes, I. Acta Math. Acad. Sci. Hungar. 2 (1951), 299-308.

2. A. Ivić, The Riemann Zeta-Function. Theory and Applications, Dover Publications, Inc. (Mineola, NY, 2003). Reprint of the 1985 original, Wiley, New York.

3. J. Karamata, Review of Freud [1]. Zentalblatt für Math. 44 (1952), 324.

4. E. Landau and A. Walfisz, Über die Nichtfortsetzbarkeit einiger durch Dichichletsche Reihen definierte Funktionen. Rend. Circ. Mat. Palermo 44 (1920), 82-86. Landau's Collected Works, Vol. 7 , Thales, Essen, 1986, 252-256.

5. H. L. Montgomery and R. C. Vaughan, Multiplicative Number Theory I: Classical Theory (Cambridge Studies in Advanced Mathematics 97), Cambridge University Press (Cambridge, 2007).

6. V. Kh. Salikhov, On the irrationality measure of $\ln 3$. Dokl. Akad. Nauk 417(6) (2007), 753-755 (Russian); Translation in Dokl. Math. 76(3) (2007), 955-957.

7. G. Tenenbaum, Introduction to Analytic and Probabilistic Number Theory (Graduate Studies in Mathematics 163), American Mathematical Society (Providence, RI, 2015).

8. Q. Wu and L. Wang, On the irrationality measure of $\log 3$. J. Number Theory 142 (2014), 264-273.

Hugh L. Montgomery,

Department of Mathematics,

University of Michigan,

Ann Arbor, MI 48109-1043,

U.S.A.

E-mail: hlm@umich.edu
Gérald Tenenbaum,

Institut Élie Cartan,

Faculté des Sciences,

Université de Lorraine,

B.P. 70239 ,

54506 Vandœuvre-lès-Nancy Cedex,

France

E-mail: gerald.tenenbaum@univ-lorraine.fr 